\title{
Psychosocial issues raised by a familial ovarian cancer register
}

\author{
Josephine Green, Frances Murton, Helen Statham
}

\begin{abstract}
A Familial Ovarian Cancer Register has been established which has recruited primarily through media publicity. In depth semi-structured interviews were carried out with 20 women who had volunteered in order to explore (1) knowledge about the disease, (2) motivations for contacting the Register, and (3) expectations. We found that interviewees were generally well informed about the symptoms of the disease as a result of their relatives' experiences. There was, nevertheless, a need for information which the Register was seen as potentially fulfilling, although most subjects gave altruistic reasons for volunteering. Only one interviewee said that the publicity about the Register had made her more anxious. Most said that their anxieties had not been affected either way by the Register. Subjects did not have a clear idea of what being on the Register would mean, although there was an expectation of screening for early signs of the disease. Many interviewees had models of familial disease which did not follow mendelian genetics. This has implications for the targetting of education and screening programmes. Other psychosocial issues raised by a register of this kind are discussed, many of which require continuing monitoring to ensure that the psychological costs do not outweigh the benefits.

( $($ Med Genet 1993;30:575-9)
\end{abstract}

With the rapid advances in human genetics, more people are going to be confronted with knowledge of their personal genetic risk. Increasingly this will involve conditions that are not 'clear cut' genetic disorders, but where the genetic contribution is rather more ambiguous. An example is familial ovarian cancer. Over 5000 women a year in the UK are diagnosed with ovarian cancer. Of these, perhaps 2 to $5 \%$ are attributable to the effects of one or more highly penetrant, dominantly inherited predisposing genes.

In September 1991 the National UK Coordinating Committee for Cancer Research (UKCCCR) Familial Ovarian Cancer Register was launched through an extensive media campaign and through contacts with doctors and articles in the medical press. The Register is targeted at women who have had two close relatives with epithelial ovarian cancer and is a research tool for genetic linkage studies and further exploration of the pathology, biology, and clinical associations of the disease.
At present, the options for action for high risk women are limited to regular check ups to monitor changes in the ovaries and prophylactic oophorectomy once childbearing is complete. It is not known whether screening will improve long term outcomes. ${ }^{1}$ Ultrasound scanning (the preferred screening method) has a very high false positive rate and many women will, therefore, be subjected to further investigations which are likely to cause anxiety. ${ }^{23}$ Prophylactic oophorectomy is, of course, not without side effects and neither is it certain that it is always effective, as there have been a few reports of apparently primary cancer arising from the peritoneum outside the ovaries in women who have had oophorectomy. Some doctors recommend oral contraception, since epidemiological data show lower rates of ovarian cancer in pill users, but this is also known to increase rates of breast cancer.

The establishment of a family cancer register is a form of population screening, that is, it is selecting from the general population a group who are thought to be at higher than average risk. In most areas of medicine the guiding principle is that such an exercise is only worthwhile for disorders that lend themselves to effective intervention. ${ }^{4}$ Indeed, some would go further and say that it is unethical unless an effective intervention can be offered. Actively seeking out healthy persons in order to tell them that they have a potentially high risk of developing a most unpleasant and usually fatal disease for which little can be done is, therefore, an unusual and controversial approach. It is, however, an essential feature of research registers of this kind. These are becoming more common, particularly for familial cancers, and use of the media is the obvious way of recruiting the necessary healthy volunteers.

Such an approach raises a number of questions, many of which cannot be answered without continuing psychosocial monitoring of the persons concerned. There are also more immediate questions which can be answered in the short term, for instance the characteristics of people who respond to this type of media publicity and their knowledge and understanding of the disease. In the case of ovarian cancer, understanding is likely to be a major problem because the concepts are actually quite complex. People have to understand that: (1) there are thought to be familial and non-familial forms of the disease; (2) fewer than $5 \%$ of cases are thought to be familial; (3) it is thought that a family with just two affected first degree relatives (and no more) occurs by chance (that is, does NOT carry the gene) in one in three cases; (4) a gene mutation is
Correspondence to Dr Green. Received 26 January 1993. Revised version accepted 1 March 1993.
Centre for Family of Cambridge,

UK.

J Green

F Murton 
involved that you either have or do not have (that is, you cannot just have it a little bit); (5) there are modifying factors, thought to be both genetic and environmental, so that even if a woman has the gene, there is still a 1 in 5 chance that she will not develop the disease; (6) the risk given to a particular woman will be in terms of her risk of developing the disease by a given age, and this will change over time; (7) men can carry the gene and pass it on to their children but will have no ill effects themselves.

It is important to be aware of the knowledge and assumptions that women bring with them to the Register so that information and services can be targeted appropriately. For example, do people assume that a family history automatically means that they are at risk or has publicity about the Register introduced this idea? How much do they know about the disease? Why have they volunteered? What are they expecting from the Register? This paper presents the findings from exploratory interviews with 20 women who had volunteered for the UKCCCR Familial Ovarian Cancer Register.

\section{Methods}

Permission was obtained from the UKCCCR for access to the Familial Ovarian Cancer Register. Twenty women, 19 early self referrals and one from a well documented family, were selected by the Register's coordinators because they lived within 100 miles of Cambridge, and asked if they would agree to be interviewed. All agreed. They were then telephoned by one of the authors and a visit arranged at a time of their choosing in their own home. All interviews were carried out by one of the authors. The Register's coordinators provided only the minimum necessary information about each interviewee and a basic family tree which gave a starting point for the interview. Interviews were semi-structured and were tape recorded with the subjects' consent. The agenda for the interview covered: demographic characteristics; general health; health behaviour; family history and history of the interviewee's involvement with the disease; knowledge and expectations of the Register; knowledge and experience of ovarian cancer screening techniques and understanding of their efficacy; social and family relationships, with particular reference to discussion of the disease and its familial links. None of the interviewees had yet been informed whether or not they were to be accepted onto the Register.

\section{Results}

SAMPLE CHARACTERISTICS

Ages ranged from 33 to 72 with the majority in their forties. Seven of the women had a relative who had died of ovarian cancer within the previous two years. Fourteen of the 20 women had had their own mother die of ovarian cancer and eight had had one or more maternal aunt affected. Seven women had had sisters affected, in three cases more than one sister. In all cases but one the putative gene would have been inherited through the maternal side of the family.

\section{ATTITUDES TO SCREENING}

Eight of the women interviewed had already had an ultrasound scan as screening for ovarian cancer. In some cases this was because they had a GP or gynaecologist who saw their family history as a risk factor, but others had had these concerns dismissed by their doctors. Some had obtained screening privately (that is, despite their doctors) and others were already involved in other ovarian cancer screening programmes. Attitudes to scanning were generally very positive, despite the physical discomfort and recognition of some of its limitations. Even when the system was clearly not working as it should (failure to recall a woman who had been told that an abnormality had been detected), the predominant feeling was that screening "has to be better than nothing". Virtually all women had regular cervical screening and regarded screening in general as part of sensible health care. There was a tendency to over-generalise from a negative cervical smear: some women thought that this indicated that they were free of any form of cancer associated with the reproductive system, and one had been told by her GP that with such a healthy looking cervix there was no need to worry about ovarian cancer.

\section{MOTIVES FOR CONTACTING THE REGISTER AND} EXPECTATIONS OF IT

Women had a variety of motives for contacting the Register. In three cases it was a need for more information about the disease, but the reason most commonly given $(n=13)$ was an altruistic desire to help with research. For some this was a way of feeling that something positive could be obtained from a personal loss, and, as such, was part of the grieving process. Many $(n=10)$ hoped that being on the Register would result in referral for ultrasound scanning or some other form of screening, but few had any clear idea of what to expect. There was a general understanding that the Register was for research purposes and that any personal benefit might be quite indirect, for example, improving the situation for their daughters or granddaughters. However, notwithstanding this altruism, there was also the feeling that, should there be any 'breakthrough', those on the Register would be the first to benefit. It was evident that a number of women had in fact already benefited from their contact with the Register simply by having had the opportunity to talk at length about their family history and concerns.

\section{ANXIETY}

On the whole, the women did not display a high level of anxiety about their own risk of ovarian cancer, although seven expressed concern for their daughters. Fourteen said that their concern about ovarian cancer being in the family had predated the publicity about the 
Register. Eight also said that they had a general fear of cancer. One woman said that the publicity about the Register had made her more anxious, two said that it had reassured them, and nine that it had made no difference. Some specific times of anxiety could be identified. Some women who were already part of a screening programme commented on the peaks and troughs of their anxiety: low immediately after a negative result but building up to a peak as the next scan became due. Another noticeable time of anxiety was as a woman approached the age at which her own mother or sister had died. Women whose mothers had died recently also showed much more anxiety about the disease, but the emphasis was on the awfulness of the disease rather than on their personal risk.

\section{PREVIOUS ASSUMPTIONS ABOUT AND}

UNDERSTANDING OF OWN RISK

All the women interviewed had some idea that ovarian cancer (or cancer more generally) was 'in the family' and assumed themselves to be, to some degree, at risk. However, this assumption owed little to an understanding that the disease could have a genetic component. Rather the dominant concept was one of 'proneness': you are assumed to be more prone to an illness that has been experienced by a close relative, particularly a close relative of the same sex. This proneness was not necessarily interpreted as a high risk but simply as a vulnerability, and did not seem to be conceptualised in numerical terms.

"It's not necessarily hereditary, but it could happen to the same members of the family because the conditions were probably the same. If the genetic conditions you had were similar to your mother, then you're more likely to get it than someone who wasn't."

Interviewees did not, on the whole, understand that only a minority of cases of the disease were thought to be familial. Indeed one asked "It is possible that some people could contract the disease without it being hereditary?". Using the same reasoning, one interviewee had refused the offer of routine breast screening on the grounds that no one in her family had ever had breast cancer and therefore she did not consider herself to be at any risk. (Few interviewees were aware of the association between breast and ovarian cancer.)

One of the most striking findings of these interviews was that phrases such as 'in the family', 'hereditary', 'inherited', and 'genetic' were frequently used in idiosyncratic ways, resulting in statements like "Well, of course, I know that hereditary things can't run in the family". People may also see a disease as familial but explain this by exposure to common risk factors: "perhaps it's because we're a farming family", rather than through inheritance. A number of women tried to comfort themselves with the thought that they were not at risk because "I take after my father's side of the family more". Few of the interviewees had realised that the disease could pass through the male line, which is consistent with the finding that in nearly all of those interviewed the gene would have come through the maternal line, but means that they did not see their brothers' daughters as potentially at risk.

\section{KNOWLEDGE OF OVARIAN CANCER}

The women interviewed were nearly all well informed about the symptoms of the disease and its treatment and prognosis. This was as a result of their personal contact with it. Nearly all had been involved in the final stages of the disease in a close relative. However, most knew nothing about known risk factors (for example, no pregnancies) or factors which are associated with lower rates of the disease, notably use of the contraceptive pill. Some women particularly drew attention to the 'blameless lifestyle' of victims of ovarian cancer, in contrast to, say, lung cancer.

A number of interviewees had a family history of other diseases as well, for example, other cancers or heart disease. The ways in which they discussed their perceived vulnerability to these other disorders shed interesting light on ways in which ovarian cancer was seen as being different. Prime among these was the fact that there are no obvious lifestyle (that is, controllable) risk factors. Those who get lung cancer should not have smoked, those with heart disease were overweight, but for those with ovarian cancer no such factors have been identified. Women therefore feel powerless in a way that, rightly or wrongly, they do not feel in the face of many other diseases.

There are two other features of ovarian cancer that were particularly upsetting to people: the relatively young age at which one can be affected, and the fact that there are no symptoms until the disease is already well established. Women were particularly conscious of this first feature if they had had a relative die who still had dependent children. The absence of symptoms may be one reason why most interviewees were so positive about screening: it was the only way that they could get early warning of a disease which they knew from personal experience was likely to prove fatal if not detected early. Actively seeking screening was, in fact, one of the two actions that these women perceived themselves as being able to take to reduce their risk. The other was to have their ovaries removed. This was not an option that had yet been considered by many of these women. Where the subject did arise during the interview, four women were in favour, four against, and two had mixed feelings. Since the interviews, at least two of the women have had an oophorectomy.

\section{WOMEN WHO DO NOT QUALIFY FOR THE} REGISTER

To qualify for the Register, documentary verification of two or more cases of epithelial ovarian cancer is needed. This is often not readily available, and the checking procedure inevitably reveals cases who do not in fact meet the criteria. Of the first 1200 self referrals to 
the Register, approximately 1000 (over $80 \%$ ) were found not to be eligible. Only two of our interviews were with women who knew that they had only one first degree relative. These women were preoccupied by fear of ovarian cancer following their mother's death. There was a need for information about the disease which they saw the Register as fulfilling. All the other interviewees believed themselves to qualify and most assumed that they would be put on the Register. Where any doubt about this arose in interview, a number of women indicated that they would be upset or disappointed: upset at the implication that they were guilty of supplying false information and disappointed to be deprived of what was seen as the main benefit of being on the Register, that is, regular screening.

\section{Discussion}

This paper has reported the findings from exploratory interviews with 20 women who volunteered for the UKCCCR Familial Ovarian Cancer Register. Such a sample size clearly does not allow us to offer any statistical analysis or definitive answers to the questions raised. Furthermore, the women that we interviewed were (with one exception) all early referrals to the Register, that is, they had responded quickly to the media publicity. As such they are probably more concerned, better informed, and more likely than other women to want both information and screening, that is, they are probably 'monitors' (information seekers). ${ }^{5}$ 'Blunters' (people whose coping strategy is to ignore or deny threatening situations) are unlikely to have referred themselves and are more likely to reach the Register through secondary referral (that is, referred via relatives or by doctors). These women are likely to be more vulnerable to any adverse effects of being on the Register, since they will be confronted with information that they had not previously considered and might prefer to avoid. Our prediction would be that later recruits to the Register, particularly secondary referrals, will be less knowledgeable and more ambivalent about the Register and about screening. Secondary referrals will also include a higher proportion of women whose risk status comes from their father's side of the family.

Over $80 \%$ of early self referrals to the Register did not meet its criteria. These women fall into three subgroups: (1) those who knew that they did not qualify; (2) those for whom it has been shown that at least one of the relatives concerned was actually affected by a different illness; (3) those who probably do have two relatives but where it is not possible to obtain documentary confirmation. Whether or not they receive any further screening will depend on the attitude of their GP and gynaecologist, or their willingness to pay to have screening carried out privately. It is to be hoped that women in groups 1 and 2 will be reassured to learn that they do not meet the criteria for being considered as 'high risk' but this cannot be assumed. On the contrary it may be that their anxiety will have been increased by the initial raised expectation of screening which remains unmet. This is particularly likely for women in group 3. It is important that people in this position should not be overlooked when registers are being evaluated.

Potentially the most important issue raised by the interviews is that of people's understanding of familial contributions to health and illness. This is an area which has received virtually no attention ${ }^{67}$ but which has considerable implications for genetic counselling as well as for health education and screening programmes. It is clear that many people do not understand the complexities involved in assessing their risk from a disease that may, or may not, have a genetic link. What beliefs do people hold about the relative contributions of hereditary, environmental, and behavioural factors? What are the implications of not sharing a geneticist's model of the transmission of the disease? One implication is that daughters of male relatives of women with ovarian cancer may not be seen as potentially at risk. Such women are therefore less likely to come forward for screening. Beliefs about causality are likely to influence attitudes to screening, as we saw with the women who refused the offer of routine mammography because no-one in her family had ever had breast cancer. With an ever increasing number of diseases (particularly cancers) being shown to have a familial component it is especially important to understand lay concepts of diseases 'running in families' in order that education and screening can be appropriately targeted.

It will soon be possible for some women to be told through linkage studies (that is, presymptomatically) that they are likely to develop ovarian cancer. This is a situation which is already being encountered for some other adult onset genetic conditions, notably Huntington's disease. Here it is being found that not everyone wants knowledge of their risk status, especially if they do not believe that the course of events can be significantly altered..$^{8-10}$ The lay 'proneness' model, referred to above, does not concern itself with mechanisms, and our interviewees showed little understanding of the concept of an ovarian cancer gene which you either have or do not have. One of the possible implications of this is that when, in the future, some of these women are told that they do not carry the gene and are therefore not at high risk, they may well not believe this information and will continue to seek reassurance through screening. In a recent report on the first family to undergo genetic linkage analysis to determine their likelihood of developing hereditary breast/ovarian cancer syndrome it was observed that "Several of the 11 individuals who were told that they were not gene carriers expressed disbelief . . . some indicated that they still wished to continue with intensive surveillance and were still considering prophylactic surgery". It is thus clear that what should be reassuring information will only be so if it is compatible with the recipient's model of the disease and its causes. Again, there are parallels with Huntington's 
disease; a recent Canadian report stated that " $10 \%$ of those given a decreased risk had psychological difficulties coping with their new status". ${ }^{12}$

In recent years we have all been urged to be responsible for our own health, to eat the right diet, take exercise, and avoid risky behaviour. The fact that there is no obvious way of following this advice to reduce one's risk of ovarian cancer is an added source of distress to at risk women. The idea that it might be genetically determined and therefore largely out of anyone's control is so contrary to the ethos of being responsible for one's own health that it would almost certainly cause even more distress were it properly understood. This is yet another reason why research programmes which identify people at risk of a serious disorder for which little can be done must be subject to psychosocial evaluation.

We gratefully acknowledge the UKCCCR for allowing us access to their Familial Ovarian Cancer Register and the contributions of Maggie Ponder and Carole Pye in selecting and contacting subjects. The Register is funded by grants from the Cancer Research Campaign and the Imperial Cancer Research Fund. Martin Richards, Maggie Ponder, and
Bruce Ponder read and improved on an earlier draft of this paper. The interviews were funded by a grant from the Cancer Research Campaign (Grant No CP 1020) awarded to Josephine Green and Maggie Ponder.

1 Creasman WT, DiSaia PJ. Screening in ovarian cancer. $\mathrm{Am}$ f Obstet Gynecol 1991;154:7-10

2 Green JM. Prenatal screening and diagnosis: some psychological and social issues. $\mathrm{Br} \mathcal{f}$ Obstet Gynaecol 1990;97:1074-6.

3 Marteau TM. Psychological costs of screening. $B M \mathcal{J}$ 1989;299:527.

4 Edwards PJ, Hall DMB. Screening, ethics and the law. BMF 1992;305:267-8.

5 Miller SM. Monitoring and blunting: validation of a questionnaire to assess styles of information seeking under threat. F Pers Soc Psychol 1987;52:345-53.

6 Davison C, Frankel S, Smith GD. Inheriting heart trouble: the relevance of common-sense ideas to preventive measures. Health Educ Res 1989;4:329-40.

7 Richards MPM Green JM Attitudes toward prenatal screening for fetal abnormality and detection of carriers of genetic disease: a discussion paper. $\mathcal{F}$ Reprod Infant Psygenetic disease: a disct

8 Craufurd D, Dodge A, Kerzin-Storrar L, Harris R. Uptake of presymptomatic predictive testing for Huntington's disease. Lancet 1989;ii:603.

9 Quaid KA, Morris M. Reluctance to undergo predictive testing: the case of Huntington disease. $A m \mathcal{F}$ Med Genet 1993;45:41-5.

10 Tibben A, Niermeijer MF, Roos RAC, et al. Understanding the low uptake of presymptomatic DNA testing for Huntington's disease. Lancet 1992;340:1416.

11 Lynch HT, Watson P. Genetic counselling and hereditary breast/ovary cancer. Lancet 1992;339:1181.

12 Huggins $M$, Bloch $M$, Wiggins $S$, et al. Predictive testing for Huntington's disease in Canada: adverse effects and unexpected results in those receiving a decreased risk. $\mathrm{Am}$ $\boldsymbol{f}$ Med Genet 1992;42:508-15. 